\title{
Implementing Relationship Education for Emerging Adult College Students: Insights from the Field
}

\author{
SPENCER B. OLMSTEAD \\ Department of Child and Family Studies, The University of Tennessee, Knoxville, \\ Tennessee, USA
}

KAY PASLEY, ANDREA S. MEYER, and PAUL S. STANFORD

Department of Family and Child Sciences, The Florida State University, Tallahassee, Florida, USA

FRANK D. FINCHAM

FSU Family Institute, The Florida State University, Tallahassee, Florida, USA

RAQUEL DELEVI

Department of Child and Family Studies, California State University at Los Angeles, Los Angeles, California, USA

With federal and state Healthy Marriage Initiatives, greater attention is on education aimed at helping couples form and maintain bealthy relationships. Emerging adults attending college are one population of focus for these efforts. Here we briefly describe a relationship education intervention for this population and provide participant feedback from a recent year of the project. Overall findings were that participants perceived the program and the facilitators positively. When asked what was helpful, they identified curriculum delivery (e.g., use of movie clips) and the teaching of skills among other things. Their assessment of the intervention differed by facilitator's gender and years of experience. Implications for relationship education for emerging adults and college instructors are discussed.

KEYWORDS couple education, relationship education, marriage education, premarital education

Address correspondence to Spencer Olmstead, 1215 W. Cumberland Ave., 419 Jessie Harris Building, Knoxville, TN 37996, USA. E-mail: solmstea@utk.edu 
Much national attention has been paid to marriage education recently, in part because of funding made available through the U.S. Department of Health and Human Services, Administration for Children and Families (Ooms \& Wilson, 2004). The goal was to promote healthy marriage, and many stepped forward to develop, deliver, and evaluate a variety of relationship education programs. However, such education predated this infusion of funding (Doherty \& Anderson, 2004; Brotherson \& Duncan, 2004). Unique to these new projects was the development of programs for populations that had been previously ignored, including low-income (Kerpelman et al., 2010; Ooms \& Wilson, 2004), underserved (Skogrand, Barrios-Bell, \& Higginbotham, 2009; Stanley, Allen, Markman, Rhoades, \& Prentice, 2010), and structurally diverse families (Dion \& Hershey, 2010; Higginbotham \& Skogrand, 2010), while enhancing the credibility of existing programs for other special populations (see Gardner, Giese, \& Parrot, 2004; Nielsen, Pinsof, Rampage, Solomon, \& Goldstein, 2004).

Here we briefly describe a relationship education program specifically designed for use with emerging adults attending college and report feedback about the program and its facilitators from participants in a recent academic year. We also attend to several facilitator characteristics expected to influence participants' experiences in the intervention.

\section{WHY FOCUS ON EMERGING ADULTS?}

According to Arnett (2004), there is increased recognition of a distinct developmental stage for those between late adolescence and young adulthood, when many young people go to college (i.e., the most recent data from 2006 show that $66 \%$ of all high school students go onto college immediately; National Center for Education Statistics, 2007). Arnett (2004, 2007) argued that during this period individuals are discovering themselves, trying out different careers, forming intimate relationships, and slowly taking on more adult responsibilities, thus the label "emerging adults." Societal changes are responsible for the creation of this life stage, such as delayed marriage and parenthood, increased expectations of post secondary education, and women's changing roles. For example, the increasing age at first marriage (Pasley \& Olmstead, 2009) coupled with views that taking on adult responsibilities at a younger age constitutes relinquishment of freedoms and life possibilities, makes emerging adulthood unique from both adolescence and young adulthood.

One primary task is preparation for long-term committed relationships such as marriage (Barry, Madsen, Nelson, Carroll, \& Badger, 2009; Lanz \& Tagliabue, 2007), and today's emerging adults spend more years being single, dating, and exploring their preferred qualities in a romantic partner (Arnett, 2004, 2007). They are also conceptualizing who they are and what their life 
goals will be, as well as forming expectations about relationship roles and responsibilities (Barry et al., 2009).

Fincham, Stanley, and Rhoades (2011) argued that there are several reasons for focusing on relationship education for this population. These reasons include: (a) they are in a developmental period which represents a "reachable moment" (Ooms \& Wilson, 2004); (b) large numbers of them want to marry, are in a romantic relationship, or want to be in one; and (c) they realize that many problematic relationship behaviors begin in these early relationships (for estimates of partner violence Smith, Thompson, Tomaka, \& Buchanan, 2005). Other risky behaviors, such as "hooking up" and "friends with benefits" (Bisson \& Levine, 2009; Paul, McManus, \& Hayes, 2000), are also prevalent, and there is evidence that those in committed romantic relationships report more health benefits (e.g., less depression and binge drinking; Braithwaite, Delevi, \& Fincham, 2010). Further, Stanley and Rhoades (2009) suggested that this age group might be most responsive to the potential benefits of prevention programs.

\section{RELATIONSHIP EDUCATION FOR EMERGING ADULTS}

Intervention and prevention represent two types of treatment for relationship issues. Intervention typically occurs in the form of couple therapy to treat problems as they occur within the relationship. Prevention often includes premarital or martial education using curriculum-based programs that address common relationship problems. Historically these programs were religiously-based and targeted to middle-class Caucasians who were engaged and expected to marry soon (Hawkins, Blanchard, Baldwin, \& Fawcett, 2008; Ooms \& Wilson, 2004). They primarily use knowledge and skills-based trainings to inform couples of typical relationship problems and educate them on strategies for relationship maintenance and enhancement (Carroll \& Doherty, 2003). Importantly, premarital education has several advantages over couple therapy, including lower cost, the potential to reach a larger and more diverse population, lower likelihood of stigmatization and fear-induction, and less intrusion into couples' lives (Larson, 2005). As a result of these advantages and the effectiveness of such programs (Fawcett, Hawkins, Blanchard, \& Carroll, 2010), a few states have provided incentives for premarital education for couples planning to marry.

Another type of recent prevention targets high school and college students who are less likely to be in romantic relationships. Evaluations of a relationship education program for adolescents demonstrated decreases in faulty relationship beliefs and increases in conflict management skills, specifically for low-income and minority youth (Kerpelman et al., 2010). For college students, the typical prevention program took the form of an undergraduate course that focused on marriage and family process, which 
tend to lack practical information on how to form and maintain healthy, committed relationships (Nielson et al., 2004). Despite the lack of systematic evaluation, these courses or programs have several strengths. First, the vast majority of the participants are not yet married, and, therefore, relationship education focuses on mate selection and exploration of potential or likely relationship pitfalls. Additionally, because emerging adults are defining self and forming perceptions of others, interventions can facilitate self-definition within the context of relating to potential partners and the development of future relationships. Although college students are an advantaged population in terms of education and income indicators, they experience multiple relationship issues (romantic and non-romantic) that are the cause of significant levels of distress (Darling, McWey, Howard, \& Olmstead, 2007).

\section{BRIEF OVERVIEW OF OUR PROGRAM}

Our program is part of a 5-year federally funded project to develop a national model of relationship education for college students through the integration of specific skills-based content into an existing, university-wide course that fulfills a social science requirement. Students attend weekly 2 hours of lecture and 1 hour of small-group work where a manualized curriculum complements and extends the content of the lectures, including a major focus on skills-based training. Thus, participants receive a 3 -hour weekly dosage of the intervention for 13 weeks of a 16-week semester. We are currently in the final year of the project. By project end, about 10,500 students will have participated in the intervention

Unique to the intervention is the small-group focus where a number of research-based topics are addressed with the goal of (a) increasing self and relationship awareness and (b) building relationship skills. For example, exploration of participants' self-awareness focuses on understanding one's family background patterns, beliefs about gender, sensitivity to personal and partner's personality, and visions about future long-term relationships. Relationship awareness entails discussions of partner selection, using selfknowledge and knowledge of others to make intentional choices, and potential barriers to fulfilling relationship expectations. Relationship skills are addressed through training in communication techniques (e.g., focused listening, speaker-listener, time-outs, conflict resolution skills) and strategies for improving and ending relationships. We target the development of skills that facilitate effective communication through practice and application during a full 7 weeks of the semester.

The initial pilot testing of the intervention resulted in revising our measures to assure full engagement with the content. For example, we broadened our language and activities to better resonate with the audience and improve implementation effectiveness (Ooms \& Wilson, 2004). Because many 
emerging adults are not in serious romantic relationships, have less relationship experience, and are more removed from marriage due to their age (Silliman \& Schumm, 2004), we targeted the relevancy of the content by talking about and using examples from committed romantic and nonromantic (e.g., friendships, roommates) relationships. We also adapted materials to better reflect the age and culture of our participants and encourage "real world" interaction with the content.

\section{Facilitator Characteristics Affecting Participant Assessment}

Most facilitators selected to deliver the skills-based portion of the intervention (small-group sessions) using the manualized curriculum are doctoral students with a master's degree in marriage and family therapy (MFT). This background offers potential skills, because clinical training focuses on active listening, empathetic responses, solicitation of answers to open-ended questions, comfort with long pauses in communication, and commenting on and facilitating group process (Ivey \& Ivey, 2003)_all skills that are valuable in working with small groups. However, this background can be detrimental if facilitators operate as if the small groups are individual or group therapy or if they lack familiarity with nonclinical research about relationship processes and the broader context of family life. Thus, they receive training in strategies for establishing clear boundaries to differentiate the class from therapy. Further, their training addresses the related body of research to enhance their responses to student queries about the topics addressed in the larger lectures and intervention protocol. Because some facilitators lack clinical experience but receive the same preparation for program delivery, we assessed whether those with MFT training and their sessions were assessed more positively by participants than those of facilitators lacking clinical training.

The facilitator's gender is also a potential factor influencing how participants experience the intervention. To our knowledge, no research has examined facilitator gender as it influences emerging adults' experiences in relationship education programs. However, research exists regarding college student ratings of course instructors based on instructors' gender. For example, some research shows that female instructors receive more positive student evaluations and that female students evaluated their female instructors more positively than they did male instructors; however, other research has failed to find a link between gender and course evaluations (see Centra \& Gaubatz, 2000, for a review).

Facilitator experience is yet another potential factor influencing participant feedback. Research has addressed the relationship between a college instructor's experience and course evaluations. Findings are that more experience is associated with better evaluations from students (e.g., McPherson, 2006; McPherson, Jewell, \& Kim, 2009). However, no study was found that 
examined the effects of facilitators' experience with relationship education on participant feedback about programs for emerging adults.

Based on this related research, we posited differences in participant ratings of the small-group sessions (the unique portion of our program) by facilitator training, gender, and experience. Specifically, we expected that those facilitators with MFT training, who were female and had more experience with the intervention, would be evaluated more positively than those without MFT training, who were males and had less experience.

This study examines participants' feedback regarding the small-group sessions from a recent academic year, because this is the unique aspect of the course. We focus on participants' assessments of the delivery of the curriculum and the facilitators with attention to facilitators' MFT background, gender, and experience with the curriculum. We also report participants' assessments of what they considered most helpful and their suggested improvements.

\section{METHODS}

Individuals who enrolled in the course give consent at the beginning of the semester and take three online surveys (beginning, mid-semester, end), or they elect to do alternative writing assignments. Because the data come from the end of the term and are anonymous, we cannot match demographic information obtained from the surveys with participant feedback. Thus, we do not know how closely our demographic summary of participant characteristics reflects those who later provide assessment data but we suspect they are similar; both sources of information were taken from two semesters (Fall 2008 and Spring 2009). Data were combined to provide an overall picture of the project from these sources.

\section{Participant Demographic Characteristics}

Of the 1587 participants from the two semesters, a majority were female $(76 \%)$ and lower classmen (freshmen $=35.8 \%$, sophomores $=36.6 \%$ ) with few seniors (6.7\%). About half (49.6\%) reported being in a romantic relationship at the beginning of the semester. The majority reported as Caucasian (68.6\%), 13.7\% as African American, 11.2\% as Latino/a, and 5.2\% as other. On average, participants were 19.33 years of age $(S D=1.50)$.

\section{Assessment Items and Analysis}

Students were asked to report their level of agreement on nine items regarding the small-group sessions where the skills-focused curriculum was used. As seen in Table 1, three items addressed the content/curriculum, and four 
TABLE 1 Ranges, Means, and Standard Deviations of Evaluation Items $(N=1273)$

\begin{tabular}{|c|c|c|c|}
\hline Item & Range & Mean & SD \\
\hline The sessions & $1-5$ & & \\
\hline Challenged me to think deeply about the subject matter & & 4.37 & .77 \\
\hline Helped me to understand the subject matter & & 4.52 & .67 \\
\hline $\begin{array}{l}\text { Used instructional techniques that engaged me in the } \\
\text { subject matter }\end{array}$ & & 4.51 & .70 \\
\hline The facilitator & $1-5$ & & \\
\hline $\begin{array}{l}\text { Was concerned about whether students learned the subject } \\
\text { matter }\end{array}$ & & 4.76 & .50 \\
\hline Was enthusiastic about the subject matter & & 4.78 & .50 \\
\hline Was enthusiastic about teaching this session & & 4.78 & .51 \\
\hline Expressed ideas clearly & & 4.74 & .53 \\
\hline Overall & $1-5$ & & \\
\hline $\begin{array}{l}\text { Considering the content, design, and structure, the Friday } \\
\text { session was }\end{array}$ & & 4.18 & .87 \\
\hline Assessment of the instructor for the Friday sessions & & 4.64 & .67 \\
\hline
\end{tabular}

addressed the facilitators; possible responses ranged from strongly disagree (1) to strongly agree (5). Two items asked about participants' overall assessment of the sessions and the facilitator; responses ranged from poor (1) to excellent (5).

Due to high correlations among the 9 items $(r=.63$ to .84; analyses not shown, contact first author for correlation table), we created an overall satisfaction scale with the first seven items only and omitted the two overall assessment items. Results of an exploratory factor analysis (principal components with oblimin rotation), showed a strong single factor solution (eigen value $=5.40 ; 77 \%$ of variance explained) and the inter-item reliability was .94. We then used a summed score in a series of one-way analyses of variance (ANOVAs) with post-hoc analyses when appropriate to examine differences in feedback based on certain facilitator characteristics (i.e., training in marriage and family therapy, gender, years of experience with the curriculum).

Participants were also asked to respond to two open-ended questions: what was most helpful from the sessions and recommendations for improvement. An initial list was created with all responses. Next, three coders examined them to identify overarching categories and assign each response to a category. Coders then met to discuss individual coding and come to consensus when there was disagreement about a category for a response. This process occurred with both sets of responses (helpful and improvements). Because participants could offer three responses to each question, a count was made of responses, so frequencies reflect the number of times it was mentioned by students. Although many students offered more than one response, some provided only one or two responses, and still others offered no feedback at all. 


\section{RESULTS}

An examination of the overall responses of participants to the individual items (see Table 1) clearly shows that they evaluated both the content and the facilitators positively. For example, on average the participants agreed that the experience in the sessions challenged them to think deeply about the content and to understand the content and engaged them in the content. Also, facilitators were perceived as concerned, enthusiastic, and clear in their delivery. Overall they rated the content, design, and structure of the sessions, and the facilitators as very good.

\section{Participants' Overall Assessment}

The results from the ANOVAs showed that the overall assessment was negatively skewed and that some of the groups did not have equal variances when tested (Norusis, 2007). When Levene's statistic was violated, Welch's statistic was examined (Brown \& Forsythe, 1974), and when more than two groups were compared (e.g., years of experience), Dunnett T3 was used as a post-hoc analysis to correct for unequal variances (Dunnett, 1980).

\section{MFT TRAINING}

Comparing mean scores on participants' overall assessment of the intervention showed no differences between facilitators having an MFT background $(M=4.64, S D=.52)$ and those not having an MFT background $(M=4.61$, $S D=.55), F(1,1272)=.55, p=.46$.

\section{GENDER}

Levene's statistic was violated $(22.67, p \leq .001)$ for differences in overall assessment by facilitator gender. Based on Welch's statistic, female facilitators $(M=4.69, S D=.47)$ had significantly higher mean scores than did male facilitators $(M=4.57, S D=.57), F(1,1168.32)=16.63, p \leq .001$.

\section{YEARS OF EXPERIENCE}

For differences in participant satisfaction by facilitators' experience delivering the intervention (1, 2, or 3 years), Levene's statistic was again violated (20.40, $p \leq .001)$. Thus, Welch's statistic showed a significant main effect for participants satisfaction, $F(2,789.52)=16.05, p \leq .001$. Post-hoc analyses indicated that facilitators with 1 year $(M=4.54, S D=.60)$ and 2 years $(M=$ $4.61, S D=.53$ ) of experience had significantly lower mean scores compared 
to facilitators with 3 years of experience $(M=4.74, S D=.43, p \leq .001)$. There were no differences between facilitators with 1 year and 2 years of experience $(p=.24)$.

\section{Responses to Open-Ended Questions}

Students reported what they found was most helpful about the sessions and offered suggestions for improvement. Contact first author for specific frequencies.

\section{Most HeLPFul}

A series of groups emerged from their responses regarding what was most helpful. Curriculum delivery (i.e., the means by which the curriculum was disseminated) was mentioned most frequently $(n=685)$, with a great deal of variation among participants comments. Examples of frequently mentioned comments about most helpful delivery elements were the use of videos to teach concepts and skills, specific activities (e.g., drawing pictures), use of population-specific PowerPoint slides, and applicable role-play scenarios.

Teaching skills was mentioned second most frequently as being helpful (defined as communication and other relationship skills aimed at helping to create a healthy relationship; $n=514$ ). Examples of skills that they identified were the "speaker-listener technique," "XYZ statements," and learning how to take a "time-out." Participants also cited concepts/knowledge gained from being a part of the project as helpful (i.e., specific concepts, information, or facts students gleaned from the sessions; $n=451$ ). Responses about concepts/knowledge generally fell into two groups: the sessions (a) aided them in grasping the content from lectures and (b) facilitated greater insight about themselves, their current romantic relationships, or how to form a healthy relationship. Specific concepts frequently mentioned included the "Seven Principles of Smart Love" (Parrot \& Parrot, 1998) and "sliding versus deciding" (Pearson, Stanley, \& Kline, 2005).

Class structure (i.e., course and classroom logistics impacting delivery) was also frequently mentioned as helpful $(n=371)$. Comments generally centered on the smaller class sizes that allowed for more focused discussion, facilitated smaller group discussion, and increased peer interaction. Participants also identified facilitator characteristics $(n=339)$, including enthusiasm, ability to engage participants, facilitate large-group interaction, and explain the concepts and ideas thoroughly. Application of the content was also mentioned ( $n=183$ ), with comments about "communication logs" (an assignment where participants practice the skills outside of class), use of session ideas in their current relationships, and use of skills in their personal lives. Last, a few participants ( $n=141$ ) commented on class culture (i.e., the 
co-construction of the class environment). Participants pointed out that the sessions were relaxed and fun; that it was easy to participate; and that they felt their views, experiences, and opinions were respected by their peers.

\section{SUGGESTED IMPROVEMENTS}

Overall, participants offered many fewer suggestions for improvement than identifying things that were most helpful. Not surprisingly, categories that surfaced from this feedback were similar to those about helpful aspects of the intervention. Curriculum delivery was identified most frequently ( $n=254$ ), with attention given to increasing the use of movie clips and group/individual activities, decreasing the number of communication logs and role-play activities, and revising activities to help participants become more involved.

Regarding class structure $(\mathrm{n}=129)$, participants focused on logistics, such as having more or fewer sessions, having sessions on different days of the week, or the grading structure of the course. Facilitator-focused comments identified changes in teaching style, approachability in emails, and entering grades. Regarding class culture, participants suggested requiring equal contribution from all peers and increasing peer-to-peer interaction. Last, a few participants specifically mentioned eliminating any out-of-session assignments (e.g., "communication logs").

\section{DISCUSSION}

Relationship education as a means to promote later healthy romantic relationships is gaining greater attention among those working with emerging adults (Fincham et al., 2011). The purpose of this study was to provide participants' insights about a relationship education intervention designed and implemented specifically for college students.

Of note is that a majority of participants reported satisfaction with the unique aspect of the project, the small-group component, as well as with those facilitating these sessions. This overall finding was supported by the comments participants offered regarding both intervention strengths and needed improvements. Specifically, program strengths included how the curriculum was delivered and the applicability of the knowledge and skills. It is also clear that improvements could be made to help the intervention be more meaningful to participants, including attention to curriculum delivery and out of session activities (e.g., "communication logs"). In fact, current curriculum revisions are incorporating these suggestions.

Although we expected differences in participant assessments by facilitator characteristics, having an MFT background did not affect participant assessment of the sessions. It may be that being trained in group process and 
the co-construction of a safe environment are important aspects of therapy (Ivey \& Ivey, 2003) but less necessary in psychoeducational interventions such as the one studied here. It may also be that the initial and ongoing training that all facilitators receive compensates for the lack of clinical experience. However, gender of the facilitator did affect participant ratings, such that females were more positively rated than were males. Even with statistical significance, we express caution about these findings, as the mean differences were modest. Because most participants were female, it may be that they identified more strongly with female facilitators, and, thus, they perceived the intervention more positively. Due to the anonymous nature of participants' feedback, we were unable to test this explanation directly (see section on limitations). Another potential influence was the facilitator's experience in delivering the intervention. As expected, those with more experience with the curriculum were rated more positively by the participants; however, we again point out that the mean differences were modest.

\section{Limitations}

The findings of this study should be considered with caution because (a) specific demographic data was linked only to general participants of the project and not necessarily those providing intervention feedback; (b) we chose only to examine a few facilitator characteristics and ignored others (e.g., age, race, or other background characteristics); and (c) our data provide only a snapshot of participant experiences from one year of the intervention. The extent to which participant feedback meaningfully resulted in intervention revision and feedback from future participants is not addressed. However, data are currently being collected to determine how important curriculum revisions, prompted by the findings of this study, have positively or negatively affected the participant perceptions of the intervention.

\section{Implications}

Our findings have important implications for the dissemination of relationship education for emerging adults in college. For example, our findings suggest the use of a variety of strategies and techniques for delivering the intervention, such that the content resonates with the participants. We found that using a variety of movie clips, hands-on activities, both small- and largegroup discussion, and using participants' own language was perceived as helpful. The responses to the open-ended questions also point to the difficulty in addressing the needs of all involved. Although feedback overall was quite positive, some participants were vocal about the limited knowledge and skill gained in the intervention. Addressing each of these is daunting and requires constant attentiveness to ensure applicability to real life experiences. 
For college instructors interested in adding a relationship component to their curriculum, we emphasize the importance of tailoring the content to the changing realities of the college student, emerging adult population. Because their romantic relationships are in constant flux as they prepare for long-term relationships and some are not in relationships, finding ways to apply the material to the diverse needs of students is essential. We also emphasize the inclusion of communication skills building. We believe that much is gained from including practice of these skills, if students are to apply tangibly what is being taught. Importantly, when delivering relationship education to emerging adult college students, facilitator characteristics of potential influence (e.g., clinical background, gender, and years of experience) may be of little concern if adequate initial and ongoing training are provided.

\section{REFERENCES}

Arnett, J. (2004). Emerging adulthood. New York, NY: Oxford University Press.

Arnett, J. (2007). Emerging adulthood: What is it, and what is it good for? Child Development Perspectives, 1, 68-73.

Barry, C., Madsen, S., Nelson, L., Carroll, J., \& Badger, S. (2009). Friendship and romantic relationship qualities in emerging adulthood: Differential associations with identity development and achieved adulthood criteria. Journal of Adult Development, 16, 209-222.

Bisson, M. A., \& Levine, T. R. (2009). Negotiating a friends with benefits relationship. Archives of Sexual Behavior, 38, 66-73.

Braithwaite, S., Delevi, R., \& Fincham, F. D. (2010). Romantic relationships and physical and mental health of college students. Personal Relationships, 17, 1-12.

Brotherson, S. E., \& Duncan, W. C. (2004). Rebinding the ties that bind: Government efforts to preserve and promote marriage. Family Relations, 53, 459-468.

Brown, M. B., \& Forsythe, A. B. (1974). The small sample behavior of some statistics which test the equality of several means. Technometrics, 16, 129-132.

Carroll, J. S., \& Doherty, W. (2003). Evaluating the effectiveness of premarital prevention programs: A meta-analytic review of outcome research. Family Relations, 52, 105-118.

Centra, J. A., \& Gaubatz, N. B. (2000). Is there gender bias in student evaluations of teaching? The Journal of Higher Education, 71, 17-33.

Darling, C. A., McWey, L. M., Howard, S. N., \& Olmstead, S. B. (2007). College student stress: The influence of interpersonal relationships on sense of coherence. Stress and Health, 23, 215-229.

Dion, M. R., \& Hershey, A. M. (2010). Relationship education for unmarried couples with children: Parental responses to the Building Strong Families Project. Journal of Couple and Relationship Therapy, 9, 161-180.

Doherty, W. J., \& Anderson, J. R. (2004). Community marriage initiatives. Family Relations, 53, 425-432.

Dunnett, C. W. (1980). Pairwise multiple comparisons in the unequal variance case. Journal of the American Statistical Association, 75, 796-800. 
Fawcett, E. B., Hawkins, A. J., Blanchard, V. L., \& Carroll, J. S. (2010). Do premarital programs really work? A meta-analytic study. Family Relations, 59, 232239.

Fincham, F. D., Stanley, S. M., \& Rhoades, G. K. (2011). Relationship education in emerging adulthood: Problems and prospects. In F. D. Fincham \& M. Cui (Eds.), Romantic relationships in emerging adulthood (pp. 293-316). Cambridge, MA: Cambridge University Press.

Gardner, S. P., Giese, K., \& Parrott, S. M. (2004). Evaluation of connections: Relationship and marriage curriculum. Family Relations, 53, 521-527.

Hawkins, A. J., Blanchard, V. L., Baldwin, S. A., \& Fawcett, E. B. (2008). Does marriage and relationship education work? A meta-analytic study. Journal of Consulting and Clinical Psychology, 76, 723-734.

Higginbotham, B. J., \& Skogrand, L. (2010). Relationship education with both married and unmarried stepcouples: An exploratory study. Journal of Couple and Relationship Therapy, 9, 133-148.

Ivey, A. E., \& Ivey, M. B. (2003). Intentional interviewing and counseling. Pacific Grove, CA: Brooks/Cole.

Kerpelman, J. L., Pittman, J. F., Adler-Baeder, F., Stringer, K. J., Eryigit, S., Cadely, H. S., \& Harrell-Levy, M. K. (2010). What adolescents bring to and learn from relationship education classes: Does social address matter? Journal of Couple and Relationship Therapy, 9, 95-112.

Lanz, M., \& Tagliabue, S. (2007). Do I really need someone in order to become an adult? Romantic relationships during emerging adulthood in Italy. Journal of Adolescent Research, 22, 531-549.

Larson, J. H. (2005). Innovations in marriage education: Introduction and challenges. Family Relations, 53, 421-424.

McPherson, M. A. (2006). Determinants of how students evaluate teachers. Journal of Economic Education, 37, 3-20.

McPherson, M. A., Jewell, R. T., \& Kim, M. (2009). What determines student evaluation scores? A random effects analysis of undergraduate economics classes. Eastern Economic Journal, 35, 37-51.

National Center for Education Statistics. (2007). Table 191: College enrollment and enrollment rates of recent high school completers, by sex: 1960 through 2006. Digest of Education Statistics. Retrieved from http://nces.ed.gov/programs/ digest/d07/tables/dt07_191.asp

Nielsen, A., Pinsof, W., Rampage, C., Solomon, A. H., \& Goldstein, S. (2004). Marriage 101: An integrated academic and experiential undergraduate marriage education course. Family Relations, 53, 485-494.

Norusis, M. J. (2007). SPSS 15.0 guide to data analysis. Englewood Cliffs, NJ: Prentice Hall.

Ooms, T., \& Wilson, P. (2004). The challenges of offering relationship and marriage education to low-income populations. Family Relations, 53, 440-447.

Parrott, L., \& Parrott, L. (1998). Relationships workbook. Grand Rapids, MI: Zondervan.

Pasley, K., \& Olmstead, S. B. (2009). Changing landscape of American family life. In J. H. Bray \& M. Stanton (Eds.), Handbook of family psychology (pp. 53-67). Boston, MA: Blackwell. 
Paul, E. L., McManus, B., \& Hayes, A. (2000). "Hookups": Characteristics and correlates of college students' spontaneous and anonymous sexual experiences. Journal of Sex Research, 37, 76-88.

Pearson, M. E., Stanley, S. M., \& Kline, G. H. (2005). Within My Reach: Instructor's manual. Denver, CO: PREP, Inc.

Silliman, B., \& Schumm, W. R. (2004). Adolescents' perceptions of marriage and premarital couples education. Family Relations, 53, 513-520.

Skogrand, L., Barrios-Bell, A., \& Higginbotham, B. (2009). Stepfamily education for Latino families: Implications for practice. Journal of Couple and Relationship Therapy, 8, 113-128.

Smith, B.A., Thompson, S., Tomaka, J., \& Buchanan, A. C. (2005). Development of the Intimate Partner Violence Attitude Scales (IPVAS) with a predominantly Mexican American college sample. Hispanic Journal of Behavioral Sciences, 27, 442-454.

Stanley, S. M., Allen, E. S., Markman, H. J., Rhoades, G. K., \& Prentice, D. L. (2010). Decreasing divorce in U.S. army couples: Results from a randomized controlled trial using PREP for Strong Bonds. Journal of Couple and Relationship Therapy, 9, 149-160.

Stanley, S. M., \& Rhoades, G. K. (2009). Marriages at risk: Relationship formation and opportunities for relationship education. In H. Benson \& S. Callan (Eds.), What works in relationship education: Lessons from academics and service deliverers in the United States and Europe (pp. 21-44). Doha, Qatar: Doha International Institute for Family Studies and Development. 